\title{
Giant Pituitary Tumours: Experience Counts
}

Can J Neurol Sci. 2012; 39: 413

In this issue of the Journal Cusimano and colleagues present their considerable experience in the surgical management of giant pituitary tumours over a 15 year period. ${ }^{1}$ This retrospective review compares the results of different surgical techniques: the technique was selected dependent on patient factors and preference of each surgeon. Due to the individual surgeon's training, experience, surgical goals, and philosophy, even if differences in patient preferences and patient factors are taken out of the equation, comparisons can often be difficult. Since randomization of patients to different surgical techniques is difficult for a number of well known reasons, comparisons within a series of patients treated at one institution by experienced surgeons may offer a reasonable glimpse of differences between the various techniques.

The authors show that in their institution very satisfactory outcomes can be achieved with patients undergoing binasal endoscopic transsphenoidal surgery (BETS), for which the authors were early adopters and innovators of this surgical technique. Advantages include a shorter median length of stay, a better degree of tumour resection and fewer overall complications compared to patients treated by other means during the same time period.

Over a 15 year period (although proportionally few tumours were operated on prior to 2002) surgical experience and even the same procedure can evolve. Looking at the median and mean follow-up, the number of patients being treated by BETS appears to have increased over the time period, which reinforces and likely reflects the satisfaction with the technique by the surgeons, guided by the successful experience/good patient outcome/ change in surgeon preference. With the caveat of the limitations identified by the authors, they have shown that excellent results can be achieved in patients selected for BETS in their institution.

\author{
Ryojo Akagami \\ University of British Columbia \\ Vancouver, BC, Canada
}

\section{REFERENCE}

1. Cusimano MD, Kan P, Nassiri F, et al. Outcomes of surgically treated giant pituitary tumours. Can J Neurol Sci. 2012;39(4): 446-57. 